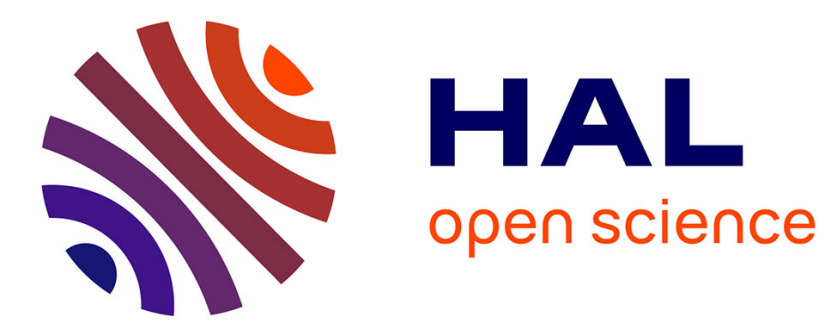

\title{
A Multi-Rate Precision Timed Programming Language for Multi-Cores
}

Alain Girault, Nicolas Hili, Éric Jenn, Eugene Yip

\section{To cite this version:}

Alain Girault, Nicolas Hili, Éric Jenn, Eugene Yip. A Multi-Rate Precision Timed Programming Language for Multi-Cores. FDL 2019 - Forum for Specification and Design Languages, Sep 2019, Southampton, United Kingdom. pp.1-8, 10.1109/FDL.2019.8876950 . hal-02399998

\section{HAL Id: hal-02399998 https://hal.inria.fr/hal-02399998}

Submitted on 9 Dec 2019

HAL is a multi-disciplinary open access archive for the deposit and dissemination of scientific research documents, whether they are published or not. The documents may come from teaching and research institutions in France or abroad, or from public or private research centers.
L'archive ouverte pluridisciplinaire HAL, est destinée au dépôt et à la diffusion de documents scientifiques de niveau recherche, publiés ou non, émanant des établissements d'enseignement et de recherche français ou étrangers, des laboratoires publics ou privés. 


\title{
A Multi-Rate Precision Timed Programming Language for Multi-Cores
}

\author{
Alain Girault*, Nicolas Hili ${ }^{\dagger}$, Eric Jenn ${ }^{\dagger \ddagger}$, and Eugene Yip ${ }^{\S}$ \\ * Univ. Grenoble Alpes, Inria, CNRS, Grenoble INP, LIG, F-38000 Grenoble, France alain.giraulteinria.fr \\ $\dagger$ IRT Saint-Exupéry, 31432, Toulouse, France, first.lasteirt-saintexupery.com \\ $\ddagger$ Thales AVS, 105 Avenue du Général Eisenhower F31036 Toulouse, France, eric.jenn@fr.thalesgroup.com \\ $\S$ Software Technologies Research Group, University of Bamberg, 96045, Germany, eugene.yip@uni-bamberg.de
}

\begin{abstract}
PREcision Timed (PRET) is a conceptual solution proposed in 2007 to address the ever increasing unpredictability of embedded processors, which results from features such as multi-level caches or deep pipelines. For many real-time systems, it is mandatory to compute a strict bound on the program's execution time. Yet, in general, computing a tight bound is extremely difficult. The rationale of PRET is to simplify both the programming language and the execution platform to allow precise execution times to be easily computed.

ForeC is a PRET programming language. It is a multithreaded variant of $\mathrm{C}$ with a synchronous execution semantics. ForeC programs are designed to be executed on multi-core processors, built around either PRET cores or classical cores. A drawback of ForeC is that programs are single rate, i.e., all reactions must be implemented to run at the fastest rate imposed by the environment. This represents a high overhead, both at design time and at run-time.

In this paper, we propose a multi-rate version of ForeC to improve its practicality and usability for industrial acceptance. We detail the syntax and semantics of the ForeC language in the context of multi-rate applications and present an implementation on a PRET multi-core architecture. Both the languages and its implementation are illustrated over a robotic application.
\end{abstract}

Index Terms-Multi-core, Precision timed, Synchronous Languages, Multi-rate, Real-time

\section{INTRODUCTION}

PREcision Timed (PRET) programming languages and architectures have been proposed in 2007 with the seminal paper from Edwards and Lee [1], to alleviate the overwhelming efforts required in computing the Worst-Case Reaction Time (WCRT) of a program. The key idea of PRET is to remove all sources of uncertainties from the architecture (e.g., cache, branch prediction, and out-of-order execution) and to provide a dedicated programming language in order to achieve predictable execution [2]. By predictable, we mean that not only the computed WCRT will be more accurate (i.e., the difference between the computed WCRT and the actual WCRT will be small), but also that the computed WCRT will be close to the Average-Case Reaction Time (ACRT). With general purpose processors, achieving this is close to impossible, due to the over-approximations necessary to provide a guaranteed WCRT [3] in presence of the architecture features mentioned

This work has been partially supported by the French Research Agency (ANR) and by the industrial partners of IRT Saint-Exupéry Scientific Cooperation Foundation (FCS). We wish to thank the anonymous reviewers for their valuable feedback. above (caches and so on), which all share the common goal of improving the ACRT.

Since 2007, several other programming languages and architectures have been proposed, notably ARPRET [4], FlexPRET [5] for the architectures, and PRET-C [6], [7] and ForeC [8]-[10] for the programming languages. In short, PRET-C and ForeC are an extension of $\mathrm{C}$ with high-level concurrency and timing instructions inspired from Esterel [11], [12]. Esterel, PRET-C, and ForeC are examples of synchronous languages, which provide an abstract, ideal, and (mathematically) tractable view of time that allows programmers to focus on the logical timing of computations, rather than their physical timing due to implementation choices. With this notion of logical time, the programming, compilation, debugging, and verification of systems is greatly simplified.

Esterel and PRET-C are multi-threaded, but their parallelism is "compiled away" by the compiler, which computes an interleaving of the threads compatible with the control dependencies expressed in the program, and finally produces sequential code meant to be executed on single-core processors (just like Esterel's compiler). In this sense, the multi-threading offered by PRET-C is a parallelism of expression. In contrast, ForeC programs are truly multi-threaded and are meant to be executed on multi-core processors.

ForeC is missing the ability to design applications that run at different rates, a feature needed to address most non-trivial control systems that mix frequent-but short-computations with infrequent-but long-executions. Multi-rate applications could be emulated by splitting long computations into smaller chunks that fit within the period of the unique rate. However, this solution is impractical because it tightly couples the structural and temporal aspects of the design. In practice, this means that a structural modification implies a temporal modification, and vice-versa, and should clearly be avoided. Moreover, the computation's logical behaviour is altered because its inputs and outputs are updated too early at the boundaries of each chunk. The solution is to integrate the ability to handle multiple rates into the programming language.

Several synchronous programming languages do offer this possibility, either as high-level language constructs, e.g., Lustre++ [13], Prelude [14], Multi-Clock Esterel [15], Simulink [16], or Giotto [17] (the last two are not strictly synchronous languages, but still relevant), or by automatic parallelization tools, e.g., [18] or [19]. The Berkeley PRET 
language also offers this possibility but with low-level instructions (the deadline instruction, noted deadi). The goal of this paper is to bring multi-rate support to ForeC, such that the semantics of Multi-Rate ForeC is backwards compatible with classical ForeC, i.e., a classical ForeC program is simply a Multi-Rate ForeC program with all rates being equal. An in-depth description of classical ForeC is given in [10].

The outline of the paper is as follows. In Section II we introduce shortly the ForeC predictable programming language. In Section III we discuss in detail the support for multiple rates in ForeC programs-our core contribution of the paper-and Section IV presents an implementation. Finally, we conclude and propose future work directions in Section V.

\section{FOREC IN A NUTSHELL}

A ForeC program is composed of a set of threads that are forked during the execution of par statements. The thread that executes the par statement is the parent of the child threads created by the statement. Threads communicate with each other via variables. Variables can be of four kinds. Input (resp. output) variables are variables sampled from (resp. emitted to) the environment. They are declared at the top-level of the program and are accessible by all threads. Variables declared with the shared keyword are shared between a parent thread and its (nested) children. Regular C variables can also be used. A ForeC program is composed of two files, a forec file that contains the program (e.g., declaration of the global variables and definition of the threads) and a foreh file that contains deployment data (e.g., declaration of the hardware platform and mapping of the threads to the platform's cores).

A ForeC program executes at the cadence of the global tick. At the beginning of the global tick, all input variables are sampled from the environment. Then, each thread executes its local tick, which consists in: (1) creating local copies of shared variables it will access, (2) performing its operations, and (3) propagating its copies of shared variables to its parent thread. Operations are performed on local copies of shared variables to avoid concurrent accesses and race conditions. If multiple threads update the same shared variable, a combination function must be specified to combine the values computed by the threads. A combination function must be associative and commutative, so that the combination is order-independent (this mechanism is inspired from Esterel's combine functions). The par statement is used to fork-join threads. A thread's local tick ends when it reaches a pause statement. A global tick ends when all local ticks end, at which point the output variables are emitted to the environment.

Fig. 1 depicts a sequence of global ticks in the case of two ForeC threads $t 1$ and $t 2$. Each blue part is the body of a tick;

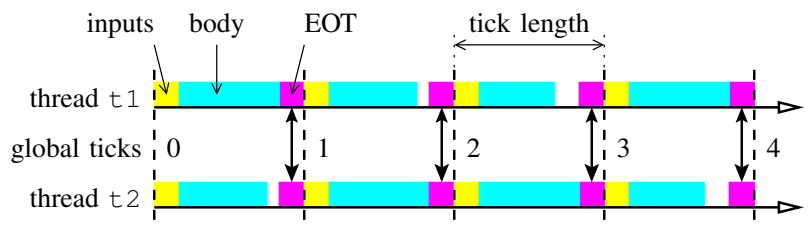

Fig. 1: The sequence of global ticks. the computations of each thread. Each yellow part samples the inputs from the environment and updates the input global variables of the thread accordingly. Finally, each magenta part is the end of tick (EOT), where the shared variables are resolved by calling their combine function and propagating their resolution back to the threads that use them, and the output global variables are emitted to the environment.

Any implementation of a ForeC program that conforms to the ForeC semantics [10] ensures that all observable input/output behaviours are invariant to implementation choices, e.g., thread scheduling or thread-to-core mapping. The way ForeC threads are actually executed in parallel depends on the chosen hardware platform. ForeC currently supports three architectures: two bare metal platforms (Xilinx multi-core MicroBlaze [20] and PTARM single-core multi-threaded PRET [21]), and x86 platforms implementing POSIX threads. When static timing analysis is of interest, the programmer can provide a static thread-to-core mapping to specify how ForeC threads are deployed on the architecture. The term core can designate a physical core (e.g., of the MicroBlaze architecture), or physical and logical threads (for PTARM and x86).

\section{Running Example: Autonomous Robot}

We demonstrate the concepts of multi-rate on a robotic demonstrator whose primary function is to guide visitors from the reception desk to the office of their host or to a meeting room. Given the map of the building and a mission specified as a set of waypoints, the robot's main objective is to move autonomously from its initial location to its target location.

Fig. 2 shows the main functions of the robot. Green circles denote sensors. Green rectangles denote internal functions and have no direct interface with the external world. Purple rectangles denote functions that interface directly with the external world via sensors and actuators, and have short reaction times of $10 \mu \mathrm{s}$. Each function output is annotated with the rate at which new data should be produced. The dashed rectangles delimit the functions that were considered for this paper.

For simplicity, we assume that the robot moves around on a plane. A localization function (LOC) is responsible for collecting information about the pose of the robot from various sources (odometry, IMU sensor, ultra-wideband (UWB) radiolocalization device, marker-based positioning device), and to compute the actual pose $(\mathrm{x}, \mathrm{y}, \theta)$ of the robot. The odometry function provides odometry data. From the robot's position, the next target position (called set point) is computed by the target generation (TG) function, and the linear and rotational speed command $(v, w)$ of the robot is elaborated by the tracking function (TRA). A speed limiter function (SPL) limits the robot's speed based on the safety monitoring of the robot's position (POM), attitude (ATM), acceleration (ACM) for collision detection, and speed (SPM). The robot can also adapt its speed when an obstacle is detected (functions ATG, LIM, OBD, SPC, and TM). Finally, the motor control (MOC) function translates the speed command $(v, w)_{S P L}$ from the speed limiter function into duty cycles $\left(d c_{L}, d c_{R}\right)$ for the 


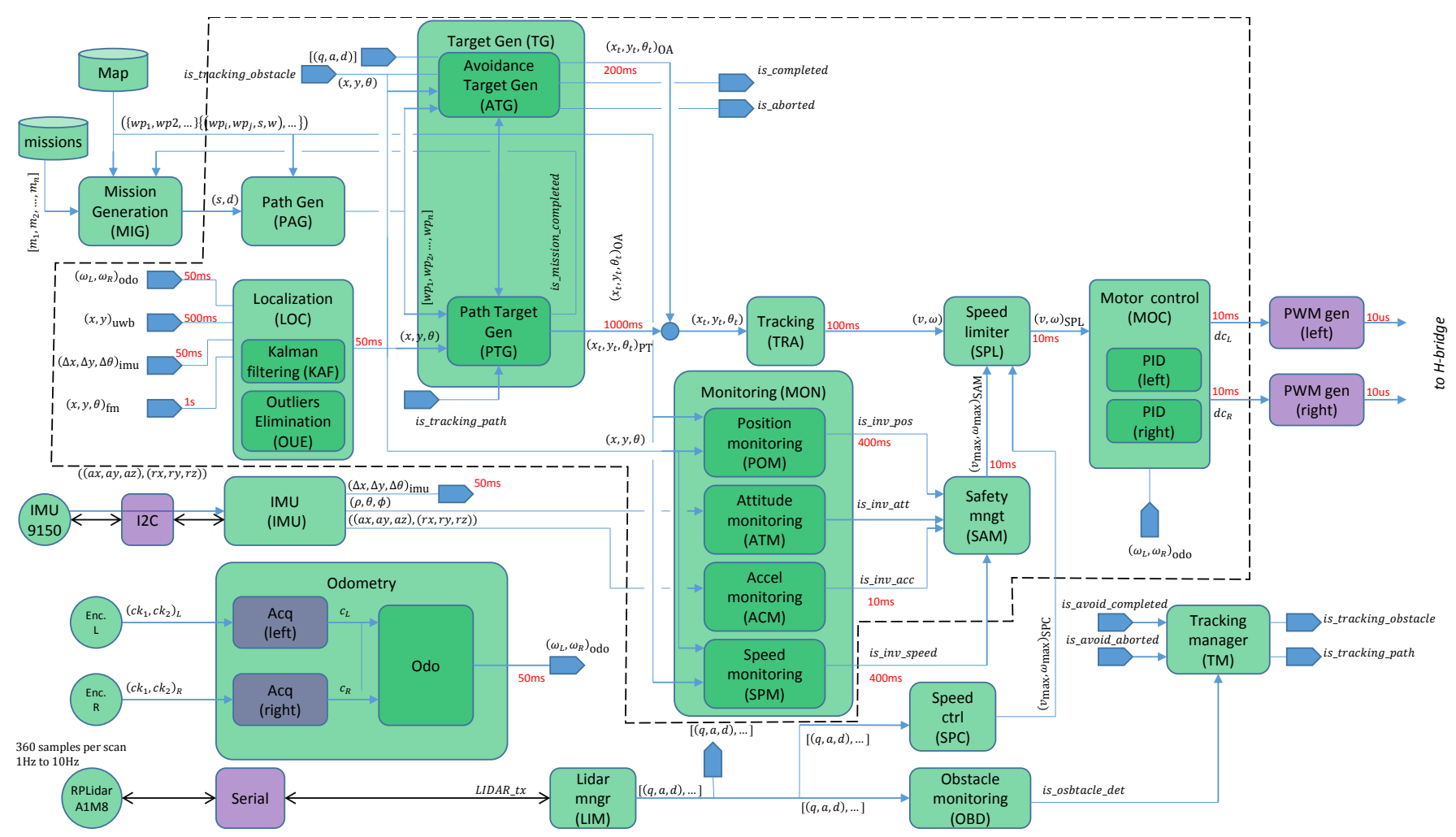

Fig. 2: Robot case study's main functions.

Pulse-Width Modulation (PWM) signals that will drive the wheel's two motors.

This example shows different cases where multi-rate is needed. First, the period of each function may depend on the actual computation of the function, or on timing constraints from the sensors and actuators the system uses to monitor its surrounding environment. For example, the period of a function collecting data from a sensor depends on the update rates of the sensor and not on the actual computation time the function requires. Various sensors and actuators may have very different timing constraints (e.g., the marker-based positioning device has an update rate of $1 s$ while a PWM signal is updated every $10 \mu \mathrm{s}$ ). Multi-rate threads is a solution to deal with these strict and inherently heterogeneous timing constraints.

Second, the rate at which a function needs to update its outputs actually depends on functional and safety constraints. For example, the attitude computed by the ATM function (to detect falls) must be updated faster than the position monitoring issued by the POM function (in order to detect that the robot does not deviate from its trajectory).

To illustrate the problem of having a mono-rate ForeC program, consider the forec file in Listing 1 that has the toplevel thread main with two operating modes. Under normal mode, the robot adjusts its linear and angular speed to follow a path. When a safety monitoring flag is raised, the abort statement is triggered, forcing the child threads to terminate and join. The robot enters the safety mode and the motors are stopped $\left(\mathrm{dc}_{\mathrm{L}}=0 ; \mathrm{dc}_{\mathrm{R}}=0 ;\right)$ until the two safety monitoring flags are cleared.
In mono-rate ForeC, the top-level thread main and all threads forked by a par statement must execute at the same rate, i.e., the rate of the slowest or fastest thread. Two problems arise if the slowest rate (e.g., thread TG) is chosen: first, when the robot is tracking an obstacle, any new avoidance set point produced by the avoidance target gen (ATG) thread would only be read by the tracking (TRA) thread every $1,000 \mathrm{~ms}$ instead of $200 \mathrm{~ms}$. Second, when a safety monitoring flag is raised, the time needed to abort the execution and to stop the motors is incompatible with the update rate of each motor's PWM signal. If the fastest rate is chosen, the slower threads would contribute a higher execution load. In the sequel, we discuss the formalization and implementation of Multi-Rate ForeC.

\section{Multi-Rate Support}

Multi-rate ForeC extends ForeC [9] with periodic clocks that we call rates (so as not to make the confusion with the richer notion of clocks in dataflow synchronous programming languages). In order to separate design details from implementation details, logical rates and concrete rates are defined in two different files. In the forec file, rates are purely logical and only upsampling and downsampling relationships can be expressed. Whenever a thread is forked (by a par statement), its default rate is that of its parent, but a different logical rate may be specified. The top-level main thread is a special case because it is not forked by a par statement; it must therefore be declared with a logical rate.

In the foreh file, the programmer assigns a concrete value for the period of the main thread's rate. Based on 
this concrete rate and on the upsampling and downsampling relationships, a concrete value can then be deduced for all the rates in the program.

\section{A. Declaration of Logical Rates}

Logical rates are declared in the forec file as C constants, with the keyword rate. For instance:

const rate $r 0$;

Upsampling and downsampling relationships are then declared in the following way:

const rate $r 1=r 0 / 2, r 2=r 0, r 3=r 1 \star 4, r 4=r 2 / 3$;

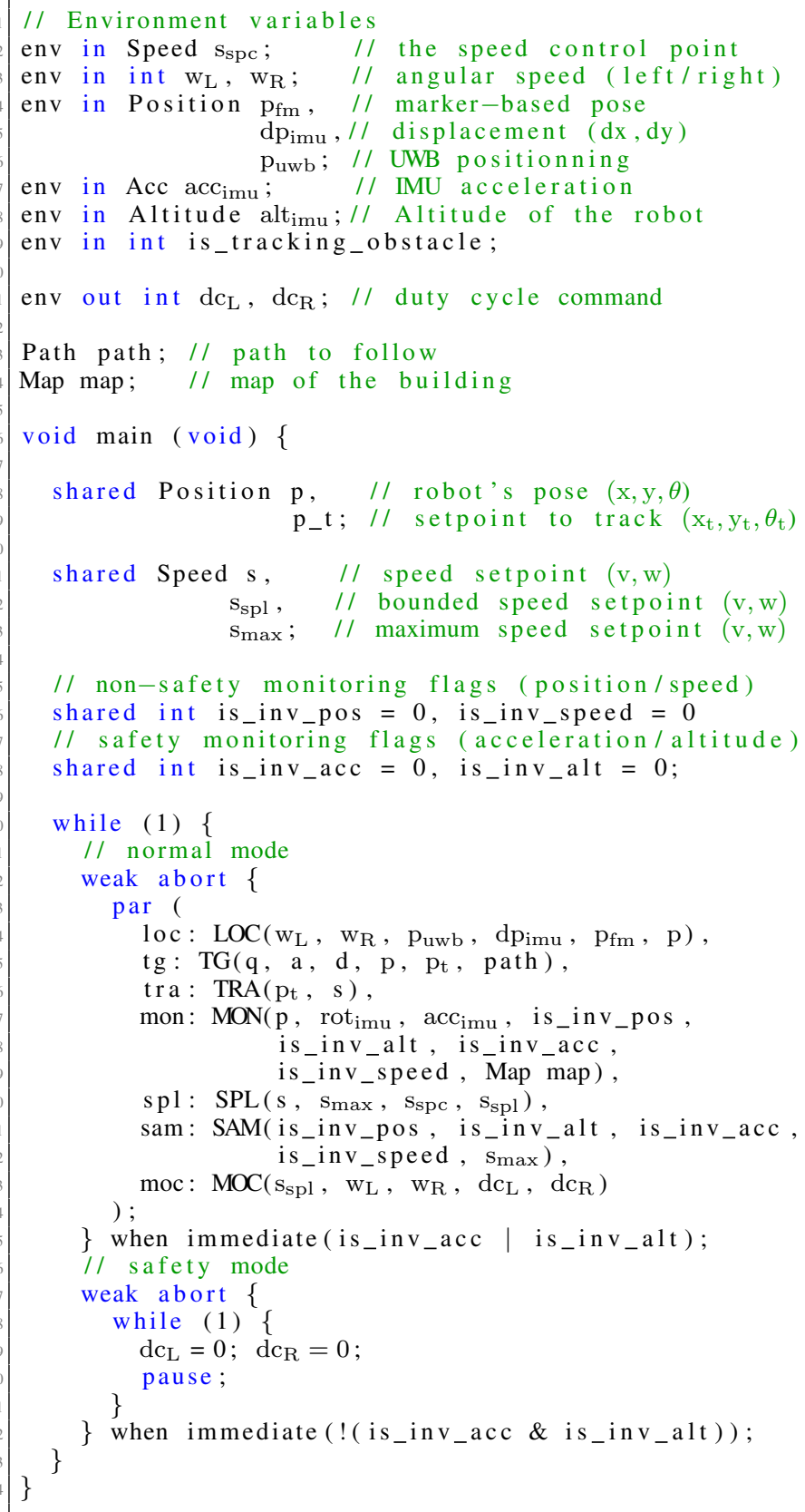

Listing 1: Mono-rate ForeC program of the autonomous robot.
The expression attached to each logical rate declaration is built using the following grammar:

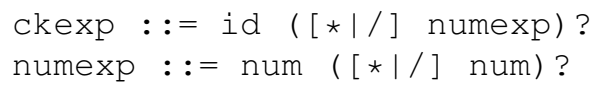

where id must be the identifier of a previously declared logical rate, and num is a strictly positive integer. In the above example, $r 1$ is two times faster than $r 0, r 2$ is identical to $r 0, r 3$ is four times slower than $r 1$, and $r 4$ is three times faster than $r 2$. In the abstract syntax tree we keep the symbolic arithmetic expression of each rate (e.g., $r 2 / 3$ for $r 4)$.

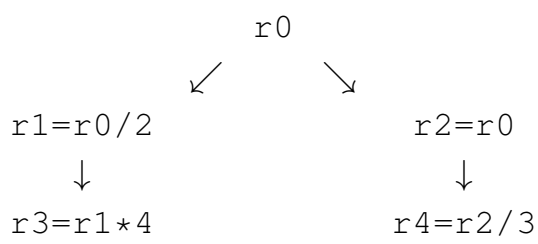

Fig. 3: The tree of logical rates for $r 0$ to $r 4$.

It follows that the structure induced by these rate relationships is a tree, as shown in Fig. 3 for the above rate declarations. Two remarks are in order:

1) The structure linking the logical rates is inferred from the rate declarations and not from the call tree of the ForeC program (i.e., not from the par statements).

2) In theory, the logical rate structure could be a forest with at least two disjoint trees; for instance if the forec file contained more than one declaration like that of $r 0$ above. We argue that this does not make sense from a programming point of view. Indeed, if the logical rate structure is a forest, then it is as if the ForeC program was actually composed of at least two disjoint ForeC programs, which communicate only via environmental variables. For this reason, we assume here on that the rate structure is a tree. This property is enforced by the ForeC compiler.

\section{B. Assignment of Concrete Rates to Threads}

Each thread either inherits the logical rate of its parent thread, or is given an explicit logical rate when it is forked, prefixed with the special character @. Since the main thread is never forked, it must be given a logical rate in its preamble, prefixed with the special character @. Listing 2 provides an example. $t 3$ inherits the rate of main when it is forked.

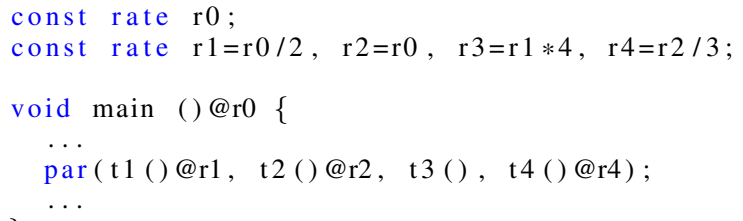

Listing 2: A simple multi-rate ForeC program.

In a well-defined ForeC program, the logical rate of any thread can therefore be expressed as a linear function of the 
logical rate of any other arbitrary thread. This property is a direct consequence of the logical rate structure: it is a tree so there is a unique path between any two nodes.

For a ForeC program to be executable, a concrete rate must be derivable for all threads. Thanks to the previous property, assigning a physical time measured in micro-seconds $(\mu s)$ to an arbitrarily selected rate (actually to its period) will allow the period of all the other rates to be easily derived. The programmer must therefore provide a concrete value to one rate in the foreh file. For instance:

const rate $\mathrm{r} 0=100$;

The tree of rates of Fig. 3 can therefore be decorated with the concrete values and with the threads, as shown in Fig. 4 for the example of Listing 2. For each rate, if the computed concrete value is an integer, then we attach this value to this rate (e.g., 50 for $r 1$ ), otherwise we keep the symbolic expression (e.g., $100 / 3$ for $r 4$ ).

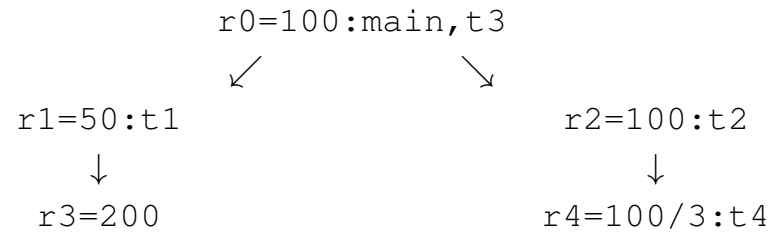

Fig. 4: The decorated tree of logical and concrete rates for Listing 2.

\section{Thread Synchronization}

In classical ForeC, all threads have the same rate, so their synchronization is straightforward: the end of a global tick (EOT) occurs when all threads end of their local tick by executing a pause. Synchronization is more complex in Multi-Rate ForeC because only the threads that end their local ticks at the same absolute time will synchronize together.

First, we distinguish between total and partial global EOT depending on whether all or only a subset of the threads participate in this global EOT. We define the counter $n_{g}$ that denotes the number of the current global tick. Second, each parallel active thread keeps track of the absolute time, in micro-seconds $(\mu s)$, which will be used to compute the time that the next (total or partial) global EOT $n_{g}$ must take place.

The time of the next global EOT, denoted $\Delta\left(n_{g}\right)$, is computed in the following way (items (1) to (5)).

(1) Let $\mathcal{A T}=\left\{t_{i}\right\}_{1 \leq i \leq k}$ be the subset of currently active parallel threads. A thread is active from the time it is forked by the par statement until the corresponding join. For each $t_{i} \in \mathcal{A T}$, let $r_{i}$ denote its concrete rate in $\mu s$, and let $s_{i}$ denote the instant of its first local tick. According to the synchrony hypothesis all computations take zero time, so in the case of a child thread, $s_{i}$ is the start of the global tick during which the par statement that forked it is executed, even if this par statement was not the first instruction of the tick. Finally, let $n_{i}$ be the counter that denotes the number of the current tick of $t_{i}$. Because the rates are different, each active thread $t_{i}$ may have a different tick count $n_{i}$.
(2) Each active thread $t_{i}$ keeps track of the time of its next local EOT $n_{i}$, denoted $\delta_{i}\left(n_{i}\right)$ and computed symbolically by Eq. (1). This equation relies on the fact that the ForeC compiler makes sure that the start time of the $n_{i}+1$ tick is equal to the end of the $n_{i}$ EOT (see Fig. 1).

$$
\delta_{i}\left(n_{i}\right)=s_{i}+n_{i} * r_{i}
$$

Computing $\delta_{i}$ symbolically avoids rounding errors. For instance, for $r_{4}=100 / 3$ we would obtain the rounded value 33. As a result, the third local EOT of $t_{4}$ (that is, $\left.\delta_{4}(3)=99\right)$ would never be equal to the first EOT of $t_{2}$ (that is, $\delta_{2}(1)=100$ )

(3) The instant $\Delta\left(n_{g}\right)$ of the next global EOT $n_{g}$ is computed by Eq. (2):

$$
\Delta\left(n_{g}\right)=\min _{t_{i} \in \mathcal{A} \mathcal{T}}\left\{\delta_{i}\left(n_{i}\right)\right\}
$$

(4) The subset $\mathcal{P} \mathcal{T}\left(n_{g}\right)$ of participating threads in this global EOT $n_{g}$ is computed by Eq. (3):

$$
\mathcal{P} \mathcal{T}\left(n_{g}\right)=\underset{t_{i} \in \mathcal{A} \mathcal{T}}{\operatorname{argmin}}\left\{\delta_{i}\left(n_{i}\right)\right\}
$$

Once this global EOT $n_{g}$ has been resolved, for each thread $t_{i} \in \mathcal{P} \mathcal{T}\left(n_{g}\right)$ we increment the tick counter $n_{i}$ and we update the value of the next local tick $\delta_{i}$. Finally, we increment the global tick counter $n_{g}$. These computations are generated by the ForeC compiler and take place in the magenta part of each tick in Fig. 1.

(5) Whenever some parent thread $t_{p}$ executes a par statement, we keep track of $t_{p}$ 's ticks by incrementing its tick counter $n_{p}$ and by computing the sequence of EOT instants $\delta_{p}\left(n_{p}\right)$ based on Eq. (1), as if $t_{p}$ was still active. These EOTs when $t_{p}$ is not active are called phantom ticks. When the join occurs (i.e., when all the threads forked by this particular par statement terminate), $t_{p}$ resumes its execution at its rate $r_{p}$, as if it had been active during the par. Thus, the next local EOT for $t_{p}$ would be $n_{p}$, which is due to occur at $\delta_{p}\left(n_{p}\right)$.

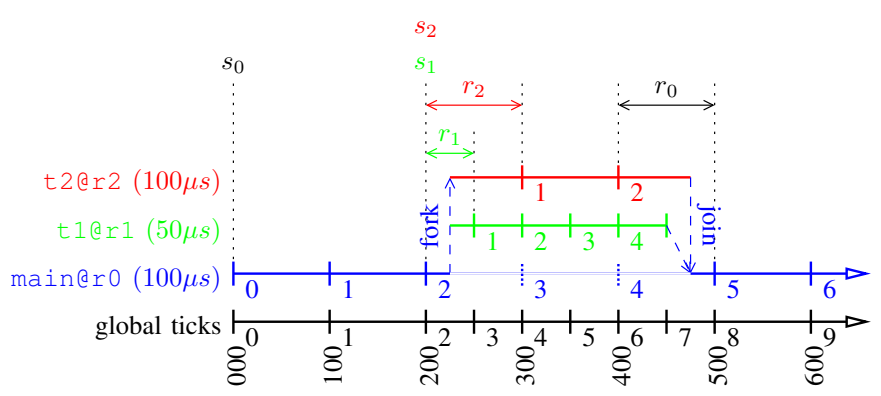

Fig. 5: A trace for a multi-rate ForeC program with three threads.

This behavior is illustrated in Fig. 5 for the example of Listing 2 by considering only threads main, $t 1$, and $t 2$. Since $r_{0}=100$, we have $\Delta(2)=\delta_{\text {main }}(2)=200$ (all units are in $\mu s$ ). In this trace, the par occurs during the global tick numbered 2, say at time 225 . From both $t 1$ and $t 2$ 's perspective, their local tick begins at $s_{1}=s_{2}=200$ (see Item (1) above). Since $r_{1}=50$, we thus have $\delta_{1}(1)=250$, and since $r_{2}=100$, we thus have $\delta_{2}(1)=300$. As a consequence, the next global EOT is partial and occurs at $\Delta(3)=250$. This 
process repeats, with the following global EOTs occurring at $\Delta(4)=300$ (total) and at $\Delta(5)=350$ (partial). In the meantime, the main thread (which is the par's parent thread) has two phantom EOTs at $\delta_{\text {main }}(3)=300$ and $\delta_{\text {main }}(4)=400$, depicted in dotted style. Then, suppose that $t 1$ terminates its execution with a pause statement, just after its EOT at 450 . Suppose also that $t 2$ terminates its execution in the middle of its tick at 475 . It follows that the join takes place at 475 , so at that time the main thread resumes its execution and its next EOT occurs at $\Delta(8)=500$.

This example highlights the benefits of Multi-Rate ForeC: t 1 and t 2 do not have the same rate, which allows them to sample inputs and emit outputs at different frequencies. This is very useful to control devices that have different dynamics and/or real-time requirements. From the point of view of the overall program (the global ticks), we see an acceleration taking place during the global ticks 3 to 8 , the rate being $50 \mu \mathrm{s}$, before resuming with the former rate of $100 \mu \mathrm{s}$.

We mention two points concerning the timing assumptions of our model. First, to ensure that threads are woken up at the required times, our bare-metal implementation (see Section IV) relies on the timing instructions offered by PRET processors [5], which allow a thread to be woken up at an absolute time. Second, compliance of a thread's execution time to the duration of its local tick is ensured by worst-case execution time (WCET) analysis [8].

Several other aspects need to be studied, e.g., the multi-rate semantics of abort statements, which, for lack of space, will be explained in a future research report.

\section{Thread Communication}

We now examine how two parallel threads having different rates communicate with each other via a shared variable. Consider Fig. 5 and the following shared variable declaration:

env out shared int $\mathrm{x}=0$ combine mod with +;

where only the copies of $x$ that have been written to (i.e., mod) are combined with mathematical addition (i.e., +). See [9], [10] for further combine policies and combine functions.

Suppose that $t 1$ and $t 2$ both increment $x$ during each of their local ticks. At $n_{g}=3$ only $t 1$ has incremented $\mathrm{x}$, so the value emitted to the environment is 1 ; this value is not propagated to $t 2$ because $t 2$ does not participate in this partial global EOT. At $n_{g}=4$, both threads have incremented $\mathrm{x}$ so the emitted value is $2+1=3$, which is propagated to both threads. The partial global EOT at $n_{g}=5$ is similar to the one at $n_{g}=3$ and the emitted value is 4 . And so on.

In Simulink, it is also possible to program communicating tasks with different rates [18]. When the sender (e.g., task A at $20 \mathrm{~ms}$ ) is faster than the receiver (e.g., task B at $80 \mathrm{~ms}$ ), a zero oracle block $\mathrm{Z}$ has to be inserted between them, which inherits the rate of $B$ and the priority of $A$ (tasks in Simulink are executed by a Real-Time Operating System with a Fixed-Priority Preemptive scheduler). In this particular case, A executes four times for every execution of $B$. As a result of the zero oracle, the value received by $B$ is the one produced by the first instance of A, as illustrated in Fig.6. Similarly, a unit delay block has to be inserted for communication from a slow to a fast task.

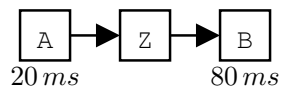

(a)

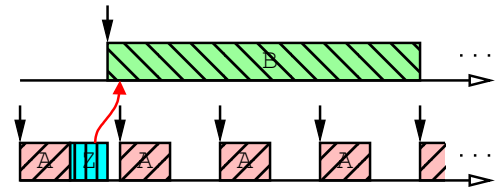

(b)
Fig. 6: Two communicating Simulink tasks.

Compared to Simulink, the advantage of ForeC's combine functions is to offer versatility in handling shared variables. Indeed, depending on the combine function chosen for the global variable shared between $A$ and $B$, the equivalent of the behavior of Fig. 6 can be achieved (if the combine function keeps the first value and ignores the three other values), but also any other behavior, be it sending the fourth value produced, the sum of the four values, their average, etc.

Finally, in Multi-Clock Esterel [15], communications between different clock zones are handled by two special devices, called the sampler and the reclocker. However, defining the semantics for sampling the presence/absence of valued signals proved difficult, so the multi-clocked emission of valued signals was left undecided. This issue does not arise with Multi-Rate ForeC because shared variables are always present throughout a program's execution. Code generation of multiclock Esterel towards multi-cores is also unsupported.

\section{E. Glue-code for Global EOTs}

In the multi-rate case, the glue code that the ForeC compiler has to generate for the global EOTs varies slightly compared to the mono-rate case. When the global EOT is total, the generated code is identical to the mono-rate case. In contrast, when the global EOT is partial, only the copies of shared variables from the threads that participate in this EOT (i.e., those in the subset $\mathcal{P} \mathcal{T}$ ) are combined.

In addition, the compiler must generate code to keep up-todate the various counters $\left(n_{g}\right.$ and, if within a par statement, $n_{p}$ and all the $n_{i}$ ), the subsets of threads $\mathcal{A T}$ and $\mathcal{P} \mathcal{T}$, the time of each thread's next local EOT (the values of $\delta_{p}, \delta_{g}$, and of all the $\delta_{i}$ ), and finally the time of the next global EOT $\Delta$. All these computations are generated by the ForeC compiler and take place within each magenta part in Fig. 1.

Regarding time predictability, the compiler checks that, for each rate $r_{i}$, the WCET between sampling the inputs on $r_{i}$ and producing the corresponding outputs is less than the concrete value of $r_{i}$. Except from the fact that this is now performed for each rate, it proceeds as in the classical ForeC compiler.

\section{BARE-MEtAl Multi-CORE PRET IMPlEMENTATion}

This section presents our implementation of Multi-Rate ForeC programs on a multi-core PRET architecture called MultiPRET [22], which consists of $n$ FlexPRET [5] cores connected via a full cross-bar for inter-core communication (see Fig. 7). FlexPRET is itself a multi-threaded single-core PRET architecture. More efficient and less resource consuming intercore communication could be provided by a time-deterministic 


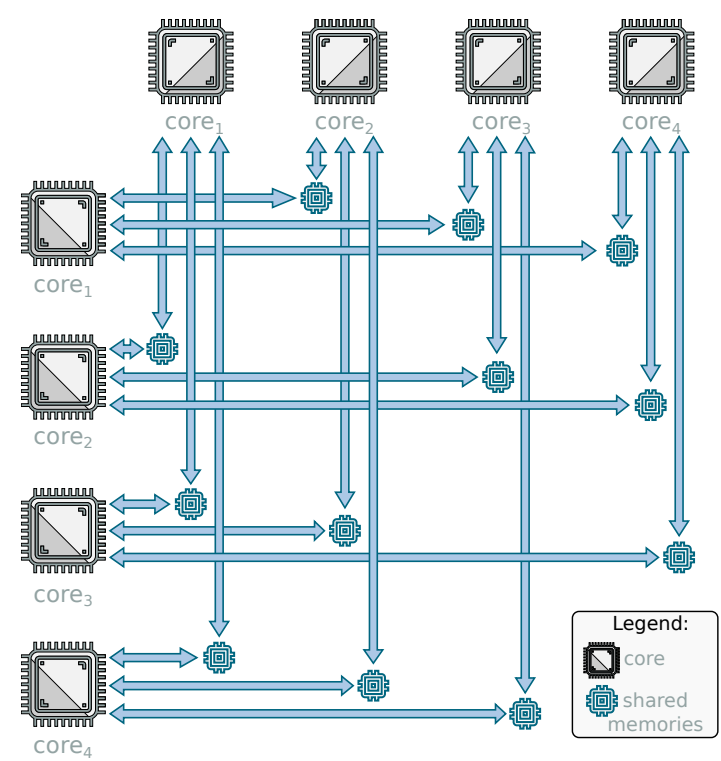

Fig. 7: MultiPRET $n$-core topology (with $n=4$ ).

bus, but for this paper we use a full cross-bar to avoid intercore interference that would be induced by bus contentions.

We adopted a full time-triggered model of execution and communication where threads can sleep or wake up at a specific (absolute) timestamp. This is guaranteed by special timing instructions provided by MultiPRET. We also assume that all environment variables are mapped to specific locations in the shared memories of the different cores. The rates at which environment variables are read or written by external processes are outside the scope of this paper. More information can be found in [22].

Fig. 8 outlines the translation of a ForeC program into a deterministic subset of $\mathrm{C}$ code that is executable on MultiPRET. The inputs of the translation is a ForeC program (a forec file) and the deployment details (a foreh file) such as the mapping of ForeC threads to the hardware threads of MultiPRET cores.

Listing 3 is an example foreh file for the ForeC program of Listing 1. It first defines the target architecture. Then, the rate of the main thread is set, which bridges the logical rates of all the ForeC threads with the physical time (in $\mu s$ ) used by the MultiPRET architecture. Finally, the deployment of each ForeC thread to a hardware thread on a core of the MultiPRET platform is defined.

Each core is uniquely identified by its ID and each ForeC

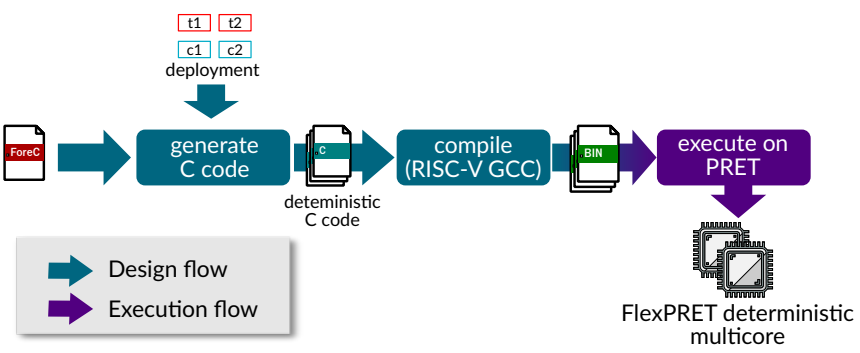

Fig. 8: Translating ForeC to C.

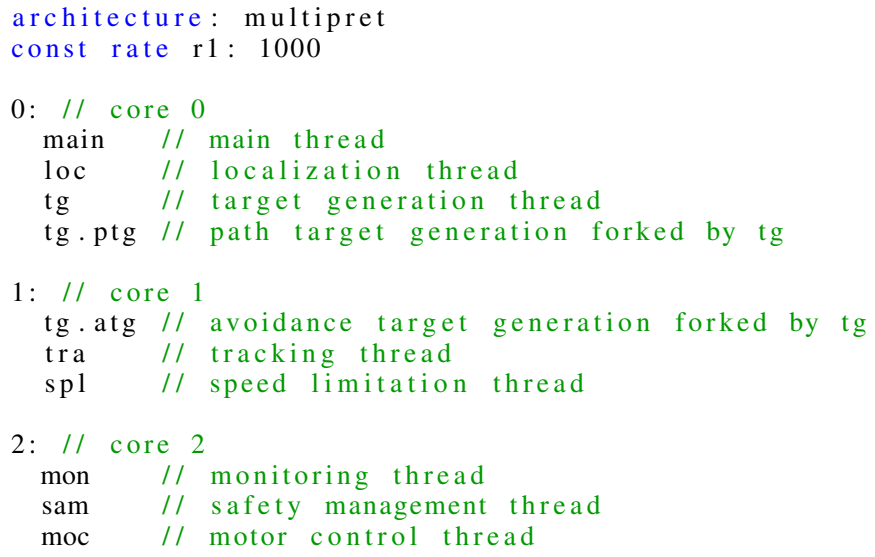

Listing 3: Example of the foreh deployment file.

thread is uniquely identified by a fully qualified name. The qualified name of the main thread is main. Direct children of the main thread are identified by their instance name (e.g., loc). Indirect children are identified by their instance name prefixed by their parent's instance name relative to main. For example, tg.ptg and tg. atg, respectively, refer to the Path target generation ( $\mathrm{PTG}$ ) thread instance and to the Avoidance target generation (ATG) thread instance forked by the Target generation $(\mathrm{TG})$ thread instance. Note that thread's instance name is used rather than the thread's name because a ForeC program may execute several instances of the same thread in parallel. For example, the PID thread has two instances left and right (cf. Fig. 2). When creating the foreh file, the developer has to ensure that all thread instances are correctly mapped to the cores of the platform.

In MultiPRET, thread-specific control instructions are used to fork threads. These instructions only affect the threads deployed on the same core as their parent. Thus, a thread deployed on core $j$ cannot fork threads or force the threads on core $k$ to join. To alleviate this problem, a technical solution was to duplicate parts of the parent thread onto the cores of their children. For example, according to Listing 3, parts of the main thread are duplicated over three cores, while parts of the tg thread are duplicated on core $_{0}$ and core $_{1}$ so that it can fork atg and ptg on their deployed core.

Listing 4 gives a excerpt of the $\mathrm{C}$ code of the localization thread LOC (as shown in Fig. 1) to be executed on core $_{0}$. Lines 1-9 define the physical addresses of all shared variables. Given the memory mapping we adopted, the two safety variables at lines 8-9 are available in the shared memory of core $_{0}$ and core $_{2}$, and thus their addresses are set to a value above $0 \times 50000000$. The LOC thread executes in a periodic loop where: i) the shared variables are copied into the local memory of the core (lines 21-29), ii) the thread performs its operations (line 31), iii) the thread waits for its period to elapse (line 33), and iv) the thread's outputs are written to the shared memory (line 34). Based on the relations between the different thread rates, the values of some shared variables may be refreshed less often than others (this is the case for 


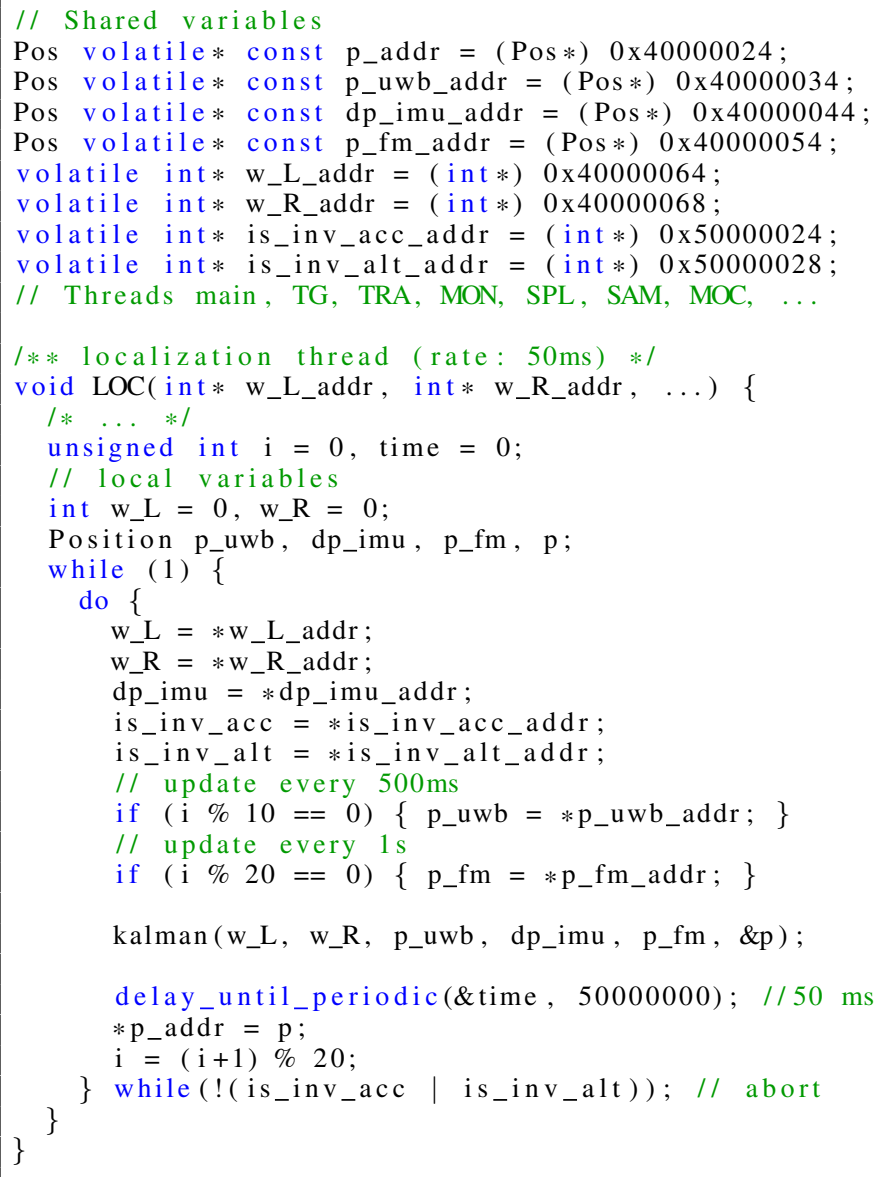

Listing 4: Localization thread deployed on core $_{0}$. Thread main and two of its child threads have been omitted.

\section{p_uwb and p_fm).}

If an abort statement encloses a par statement, then the semantics of the abort is delegated to the par's child threads. For example, the condition of the weak abort statement in Listing 1 (lines 32-45) is evaluated in Listing 4 by the localization thread LOC (lines 20-36).

\section{CONCLUSiON}

We have presented a multi-rate extension to the ForeC multi-threaded precision timed language, and have proposed elements of its implementation. By providing the capability to handle directly threads with multiple rates, Multi-Rate ForeC offers the possibility to design embedded software with multiple temporal dynamics, while guaranteeing time predictability. As a result, it reduces both the development time and execution time of actual applications, and finally, enhances the industrial acceptance of ForeC. This new capability has been illustrated by a robotic application deployed on the MultiPRET deterministic PRET processor.

Future work will include the generalisation of rates with offsets, and the support for a forest of clocks. Integration with a tool is on-going.

\section{REFERENCES}

[1] S. A. Edwards and E. A. Lee, "The Case for the Precision Timed (PRET) Machine," in Design Automation Conference (DAC). ACM, 2007, pp. 264-265.

[2] P. Axer, R. Ernst, H. Falk, A. Girault, D. Grund, N. Guan, B. Jonsson, P. Marwedel, J. Reineke, C. Rochange, M. Sebastian, R. von Hanxleden, R. Wilhelm, and W. Yi, "Building timing predictable embedded systems," ACM Transactions on Embedded Computing Systems, vol. 13, no. 4, p. 82, Feb. 2014.

[3] R. Wilhelm, J. Engblom, A. Ermedahl, N. Holsti, S. Thesing, D. Whalley, G. Bernat, C. Ferdinand, R. Heckmann, T. Mitra et al., "The WorstCase Execution-Time Problem - Overview of Methods and Survey of Tools," ACM Transactions on Embedded Computing Systems (TECS), vol. 7, no. 3, p. 36, 2008.

[4] P. S. Roop, "Predictable Reactive Processors for Next Generation Computing: A Proposal," School of Engineering Report, vol. 662, 2008.

[5] M. Zimmer, D. Broman, C. Shaver, and E. A. Lee, "FlexPRET: A Processor Platform for Mixed-Criticality Systems," in Real-Time and Embedded Technology and Applications Symposium (RTAS). IEEE, 2014, pp. 101-110.

[6] S. Andalam, P. Roop, and A. Girault, "Predictable Multithreading of Embedded Applications Using PRET-C," in International Conference on Formal Methods and Models for Codesign. IEEE, Feb. 2010.

[7] S. Andalam, P. S. Roop, A. Girault, and C. Traulsen, "A Predictable Framework for Safety-Critical Embedded Systems," IEEE Transactions on Computers, vol. 63, no. 7, pp. 1600-1612, July 2014.

[8] E. Yip, P. S. Roop, M. Biglari-Abhari, and A. Girault, "Programming and Timing Analysis of Parallel Programs on Multicores," in International Conference on Application of Concurrency to System Design (ACSD). IEEE, 2013, pp. 160-169.

[9] E. Yip, A. Girault, P. Roop, and M. Biglari-Abhari, "The ForeC synchronous deterministic parallel programming language for multicores," in International Symposium on Embedded Multicore/Many-core Systems-on-Chip (MCSoC). Lyon, France: IEEE, Oct. 2016.

[10] E. Yip, P. Roop, A. Girault, and M. Biglari-Abhari, "Synchronous deterministic parallel programming for multicores with ForeC," Inria, Research Report RR-8943, Aug. 2016. [Online]. Available: https://hal.inria.fr/hal-01351552

[11] G. Berry and G. Gonthier, "The Esterel synchronous programming language: Design, semantics, implementation," Science of Computer Programming, vol. 19, no. 2, pp. 87-152, 1992.

[12] F. Boussinot and R. de Simone, "The Esterel language," Proceedings of the IEEE, vol. 79, no. 9, pp. 1293-1304, Sep. 1991.

[13] M. Alras, P. Caspi, A. Girault, and P. Raymond, "Model-based design of embeded control systems by means of a synchronous intermediate model," in International Conference on Embedded Systems and Software (ICESS). IEEE, May 2009, pp. 3-10.

[14] J. Forget, F. Boniol, D. Lesens, and C. Pagetti, "A multi-periodic synchronous data-flow language," in High Assurance Systems Engineering Symposium (HASE). IEEE, 2008.

[15] G. Berry and E. Sentovich, "Multiclock Esterel," in IFIP Conference on Correct Hardware Design and Verification Methods, CHARME'01, ser. LNCS, vol. 2144. Springer-Verlag, Sep. 2001, pp. 110-125.

[16] The MathWorks, Inc., Real-Time Workshop User's Guide, v3, 1999.

[17] T. Henzinger, B. Horowitz, and C. Kirsch, "Giotto: A time-triggered language for embedded programming," in International Workshop on Embedded Software (EMSOFT), ser. LNCS, vol. 2211. Springer, Oct. 2001.

[18] A. Girault, X. Nicollin, and M. Pouzet, "Automatic rate desynchronization of embedded reactive programs," ACM Transactions on Embedded Computing Systems, vol. 5, no. 3, pp. 687-717, Aug. 2006.

[19] P. Caspi, N. Scaife, C. Sofronis, and S. Tripakis, "Semantics-preserving multitask implementation of synchronous programs," ACM Transactions on Embedded Computing Systems, vol. 7, no. 2, Feb. 2008.

[20] Xilinx, MicroBlaze Processor Reference Guide, 2012, accessed 28 Aug. 2019. [Online]. Available: https://www.xilinx.com/support/ documentation/sw_manuals/xilinx 13_4/mb_ref_guide.pdf

[21] I. Liu, J. Reineke, D. Broman, M. Zimmer, and E. A. Lee, "A PRET Microarchitecture Implementation with Repeatable Timing and Competitive Performance," in International Conference on Computer Design (ICCD). IEEE, Sep. 2012, pp. 87-93.

[22] N. Hili, A. Girault, and E. Jenn, "Worst-Case Reaction Time Optimization on Deterministic Multi-Core Architectures with Synchronous Languages," in International Conference on Embedded and Real-Time Computing Systems and Applications (RTCSA). IEEE, Aug. 2019. 